\title{
Resonances from lattice QCD
}

\author{
Raúl A. Briceño ${ }^{1,2, \star}$ \\ ${ }^{1}$ Thomas Jefferson National Accelerator Facility, 12000 Jefferson Avenue, Newport News, VA 23606, USA \\ ${ }^{2}$ Department of Physics, Old Dominion University, Norfolk, VA 23529, USA
}

\begin{abstract}
The spectrum of hadron is mainly composed as shortly-lived states (resonance) that decay onto two or more hadrons. These resonances play an important role in a variety of phenomenologically significant processes. In this talk, I give an overview on the present status of a rigorous program for studying of resonances and their properties using lattice QCD. I explain the formalism needed for extracting resonant amplitudes from the finite-volume spectra. From these one can extract the masses and widths of resonances. I present some recent examples that illustrate the power of these ideas. I then explain similar formalism that allows for the determination of resonant electroweak amplitudes from finite-volume matrix elements. I use the recent calculation of the $\pi \gamma^{\star} \rightarrow \pi \pi$ amplitude as an example illustrating the power of this formalism. From such amplitudes one can determine transition form factors of resonances. I close by reviewing on-going efforts to generalize these ideas to increasingly complex reactions and I then give a outlook of the field.
\end{abstract}

\section{Introduction}

Hadronic resonances are ubiquitous in nature, and as a result play an essential role in a variety of phenomenologically significant processes. One such example is the famous $\Delta I=1 / 2$ rule in $K \rightarrow \pi \pi$ weak decays. This empirical "rule" states that hadronic decay of the kaon to two pions is dominated by the process where the isospin of the final state is zero. The corresponding amplitude is approximately an order of magnitude larger than that when the $\pi \pi$ final state is projected onto an isotensor configuration. This somewhat surprising observation becomes quite natural when one remembers that the isoscalar channel contains the $\sigma$ resonance, which is nearly degenerate with the kaon, while the isotensor channel contains no resonances.

By now there is overwhelming evidence that without resonances most atomic nuclei would not be able to form. This is seen for example in the essential role played by resonances, in particular the $\sigma, \rho$, and $\omega$, in the construction of phenomenological potentials that accurately describe experimental data [1]. Another example is the well known Hoyle resonance, without which there would not be enough carbon, oxygen, nickel, etc. to support life as we know it [2].

Given that most hadrons are unstable, resonances give us a window into the mechanism responsible for binding quarks and gluons together, which in turn would give us further insight into the rules

$\star$ Thanks his colleagues and collaborators for their continuous support. RAB would also like to thank his colleagues from the Hadron Spectrum Collaboration for granting permission to share results obtained in collaboration with them. RAB acknowledges support from U.S. Department of Energy contract DE-AC05-06OR23177, under which Jefferson Science Associates, LLC, manages and operates Jefferson Lab. 
governing the fundamental theory of the strong interaction, quantum chromodynamics (QCD), at low energies. This is the motivation of a substantial experimental effort to precisely measure the basic properties of resonances (mass, decay widths, couplings, etc.) and also explore the fringes of the hadronic spectrum, where these rules can be put to a test. Prominent examples include the searches for exotic in the GlueX and COMPASS experiments, tetraquark ${ }^{1}$ and pentaquarks searches in Belle, BES and LHCb among other.

Given these and other experimental efforts, there is a strong demand for a parallel theoretical program. Here I outline a theoretical effort to have a program that satisfies the following four criteria:

- confirmation of existence: there is a long list of states that are awaiting theoretical confirmation, plenty of examples can be found in the XYZ (see Ref. [5] for a recent review),

- predict existence: presently rigorous theoretical predictions of non-conventional hadrons are absent,

- identify production/decay mechanism: in order to expedite and guide experimental searches, it would be useful to have a theoretical determination of the prominent production/decay mechanism including couplings to corresponding channel,

- structural information: there are a variety of properties that are experimentally inaccessible but are essential to gain insight into the true nature of a corresponding state, e.g., gluonic structure of hybrid mesons.

Resonances, by definition, lie above at least one open threshold. Most, in fact, lie above multiple open thresholds where two or more particles can go on-shell. In order to have a complete understanding of these resonances, one would need to have a QCD-based effort that is capable of dealing with multichannel, multiparticle systems. This is undoubtably a challenging and daunting task, but thanks to lattice QCD this is not an insurmountable challenge.

Lattice QCD efforts have largely been focused in the extraction of the spectrum and properties of QCD stable states. This is mainly due to the fact that these are the states that are most readily accessible using standard lattice QCD techniques. In fact, given the present framework of lattice QCD calculations and the nature of resonant states, it is not at all obvious that the latter can be accessed via lattice QCD. Nevertheless, there has been tremendous progress that has allowed for the exploration of resonant processes that were previously thought to be inaccessible via lattice QCD (for a recent review in the field see [6]).

Before explaining the challenges associated with study resonances on the lattice, it is necessary to begin by reviewing the definition of resonances as complex-valued poles in unphysical sheets of scattering amplitudes. I review the building principles of this in Sec. 2. In Sec. 3, I discuss the formalism for extracting two-body scattering amplitude from the finite-volume spectrum obtained from lattice QCD calculations, and I illustrate the power of these ideas in the light mesonic sector. In Sec. 4.1, I discuss extension of these ideas to extract electroweak amplitudes, focusing my attention to $1 \rightarrow 2$ processes. I present a recent example in Sec. 4.2 using the formalism discussed. Lastly, in Sec. 5 I speculate what the future holds for study of resonances on the lattice.

\section{Scattering theory and composite states}

Here I review some of the basic principles in scattering theory that are relevant for the discussion that follows. In particular, we need a rigorous definition of resonances and a prescription to obtain their information using lattice QCD. As it turns out, we can only access their informations from

\footnotetext{
${ }^{1}$ For recent lattice QCD efforts in searches for tetraquarks see Refs. [3, 4].
} 
scattering amplitude. For simplicity, I will focus my attention to scattering amplitudes where there are at most two hadrons in the initial or final states, but I will not restrict the number of two-particle channels that might be kinematically open. ${ }^{2}$

By definition, resonances are unstable under the strong interaction. In other words, they cannot be identified as asymptotic states. Because they commonly manifest themselves as dynamical enhancements in cross sections, they have been qualitatively understood as "bumps in cross sections". Although this serves as a rule of thumb, it is a terribly unsatisfying definition. Furthermore, it is not hard to find examples of resonances that do not appear as clear bumps in cross sections, e.g. the $\sigma$ and the $\kappa$. Alternatively, one can rigorously define resonances as poles in scattering amplitudes in the complex plane of the Mandelstam variable $s \equiv E^{\star 2}$. ${ }^{3}$ This definition holds for both bound states and resonances. Bound states are real-valued poles below the lowest threshold, while resonances are complex-valued poles and typically lie above the lowest threshold. ${ }^{4}$ The value of the pole location $\left(s_{R}\right)$ are related the resonance's mass $\left(m_{R}\right)$ and width $\left(\Gamma_{R}\right), \sqrt{s_{R}}=m_{R}-\frac{i}{2} \Gamma_{R}$.

At every threshold, the scattering amplitude has a square-root singularity. Let $\mathcal{M}_{\ell ; a b}$ be the $\ell$ partial wave component of the scattering amplitude for incoming $a$ and outgoing $b$ channels. For example, in Sec. 3.3 we will discuss S-wave wave scattering amplitudes where $a$ and $b$ correspond to $\pi \pi$ and $K \bar{K}$ scattering states. Having this notation, one can explicitly see this singularity by evaluating the imaginary part of the inverse of the amplitude,

$$
\operatorname{Im}\left(\mathcal{M}_{\ell}^{-1}(s)\right)_{a b}=-\delta_{a b} \frac{1}{16 \pi} \frac{2 q_{a}^{\star}}{\sqrt{s}} \Theta\left(s-s_{a}\right),
$$

where $s_{a}=\left(E_{a}^{\text {thr. }}\right)^{2}$ is the value of the ath threshold, and $q_{a} \sim \sqrt{s-s_{a}}$ is the cm relative momentum and the source of the square root singularity. This in turns results in there being $2^{N}$ Riemann sheets for $N$ open channels. Causality prohibits complex-valued poles in the physical Riemann sheet, defined by $\operatorname{Im}\left[q_{a}\right]>0 \forall i$. As a result, only real-valued poles can exist in this sheet, namely bound states. Furthermore, this tells us that resonances can only correspond to complex poles on the unphysical sheets, where at least one relative momentum satisfies $\operatorname{Im}\left[q_{a}\right]<0$.

This definition does several things for us. First, it explains why some resonances manifest themselves as bumps in cross sections. Second, it explains how one can (and perhaps "should") think of the evolution of resonances and bound states with the quark masses. If we zoom in to a given pole in the scattering amplitude and we now consider that the amplitude itself depend on the values of the quark masses, which I denote a $m_{q}$, we find

$$
\mathcal{M}_{\ell ; a b}\left(s ; m_{q}\right) \sim \frac{g_{a}\left(m_{q}\right) g_{b}\left(m_{q}\right)}{s_{R}\left(m_{q}\right)-s} .
$$

As we dial the quark masses we expect $s_{R}\left(m_{q}\right)$ and $g_{a}\left(m_{q}\right)$ to vary, but there is no reason as to why in general they might go to zero or infinity. As a result, with the quark mass we might expect poles to move in the complex plane, even change sheets, and their coupling could very well vary, but we do not expect them to evaporate. This qualitatively explains why we observe some resonances becoming bound for heavier quark masses. This also tells us that we should probably think of resonances and bound states more generically as composite states that for some range of parameters might be accidentally bound or not.

\footnotetext{
${ }^{2}$ For a pedagogical introduction to scattering theory and the theory of composite states see [7].

${ }^{3} E^{\star}$ is the center of mass (cm) energy of the system, sometimes also labeled $E_{\mathrm{cm}}$.

${ }^{4}$ Resonances in the $\ell=0$ partial wave can appear as a complex-valued pole on the second Riemann sheet below threshold.
} 


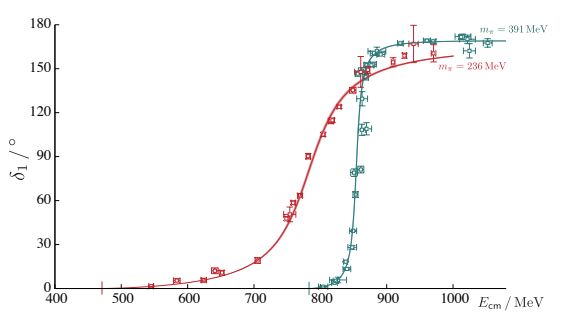

(a)

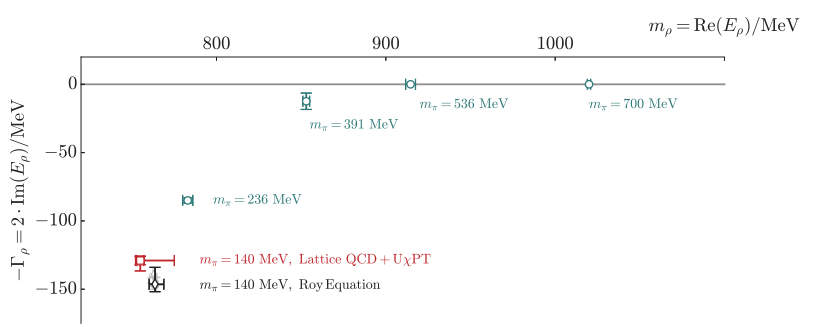

(b)

Figure 1. (a) Shown are the $\pi \pi$ isovector scattering phase shift from Refs. $[8,9]$ which correspond to approximate values of $m_{\pi}=236,391 \mathrm{MeV}$. (b) In blue are the pole position of the $\rho$ resonance for a range of values of $m_{\pi}$ from a range of lattice calculations [8-11]. Also shown in red the corresponding pole of the chiral extrapolation of the scattering amplitudes obtained in Ref. [12] and discussed in 3.2.1. These are compared to the phenomenological value of the pole position in black [13-18]

\section{Spectroscopy from lattice QCD: scattering amplitudes and resonances}

Lattice QCD calculations are necessarily performed in a finite volume. This has multiple ramifications to the theory. First, one cannot define asymptotic states and consequently one cannot readily access their information. Furthermore, this alters the analytic structure of the correlation functions. For one, there are no square root singularities. Instead, this branch cut is replaced with a tower of real-value poles corresponding to the finite-volume energies. Consequently, in a finite volume there is a single sheet, namely the physical sheet. This then implies that strictly speaking there are no resonances in a finite volume, and as a result one cannot access resonances or their properties directly.

Alternatively, one can relate information obtained using correlation functions in a finite-volume to infinite-volume scattering amplitudes. Once one has obtained the scattering amplitudes, one can proceed to obtain the resonance information. There are several ideas in the market as to how one may do this [6]. Here I focus on arguably the more formally robust ideas for extracting hadronic scattering amplitude and in Sec. 4 I discuss similar ideas for electroweak amplitudes.

\subsection{Finite-volume spectrum: extraction and interpretation}

The basic idea is to exploit the dependence of the finite-volume spectrum on the underlying dynamics of the system. To do this in a non-perturbative fashion, one must obtain a non-perturbative quantization that the spectrum must satisfy. Such a non-perturbative expression was first derived by Martin Lüscher for two identical bosons at rest [19, 20]. Since then, Lüscher's formalism has been generalized to arbitrary complex two-body systems, accommodating any number of open channels with constituents carry arbitrary intrinsic spin [21-37],

$$
\operatorname{det}\left[F^{-1}(P, L)+\mathcal{M}(s)\right]=0
$$

where $F^{-1}(P, L)$ is a known finite-volume function that depends purely on kinematics [37]. Here the determinant acts in the space of degrees of freedom of the system. For energy where only twoparticle states can go on-shell, these are just the angular momentum between the particles and the open channels. This equation is satisfied whenever $P^{0}$ coincides with a finite-volume eigenvalue, and explains how the infinite-volume scattering amplitude can be constrained at that given energy. These 


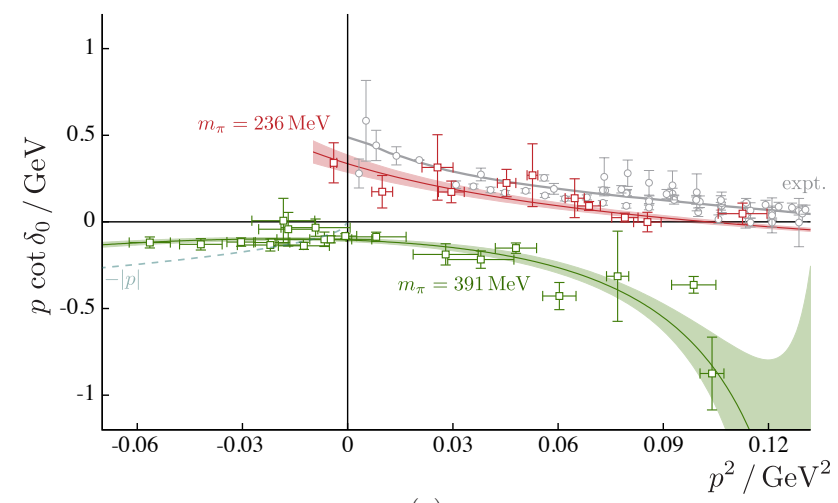

(a)

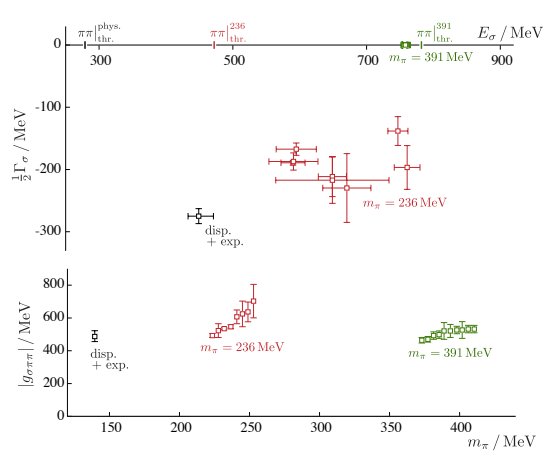

(b)

Figure 2. (a) Shown are the values of the $p \cot \delta$ from Ref. [52] compared with the phenomenologi$\mathrm{cal} /$ experimental values [17, 53-56]. (b) Top panel shows the pole location of the $\sigma$ and $\sigma \rightarrow \pi \pi$ couplings from lattice QCD [52] and dispersive analysis of scattering data [17, 57, 58].

energies can, in practice, be extracted from two-point correlation functions,

$$
C_{a b}^{2 p t .}(t, \mathbf{P}) \equiv\left\langle 0\left|O_{b}(t, \mathbf{P}) O_{a}^{\dagger}(0, \mathbf{P})\right| 0\right\rangle=\sum_{n} Z_{b, n} Z_{a, n}^{\dagger} e^{-E_{n} t}
$$

where $O_{a}^{\dagger}$ and $O_{b}$ are generic source/sink operators with the same quantum numbers.

In order to study resonances, it is necessary to map out the energy-dependence of the scattering amplitude and from it deduce its pole structure. This requires determining a large number of energy levels, not just the ground states. This is arguably the most challenging aspect of this program. Fortunately, there has been a significant effort on this front [11,38-51], which have allowed one to determine energies above multiple open threshold. In Sec. 3.3 I discuss an example of this in the isoscalar sector.

\subsection{Elastic scattering processes}

In lattice QCD calculations, this ideas have been primarily been put into practice in determining elastic scattering amplitudes. Arguably the first signal that the field is reaching a level of maturity was the study of the isovector $\pi \pi$ scattering phase shift studied in Ref. [8]. There the authors used three different volumes, multiples boosts and irreducible representations (irreps) to constrain the scattering amplitude at over 30 different energies in the elastic region. At the time, this was unprecedented achievement which was followed by other successful studies [9, 59-61]. ${ }^{5}$

Unlike the isovector $\pi \pi$ channel, until recently the isoscalar channel has been virtually unexplored. Having the same quantum numbers of the vacuum, this is one of the most phenomenologically interesting sectors of QCD. Having completely disconnected diagrams, it is also one of the more computationally challenging channels to explore via lattice QCD. Nevertheless, one given the technology briefly discussed above, it is now possible to calculate spectra in this sector. The first time this was

\footnotetext{
${ }^{5}$ Another benchmark $N_{f}=2+1$ calculation worth noting is that of [62] which predates that of Ref. [8].
} 


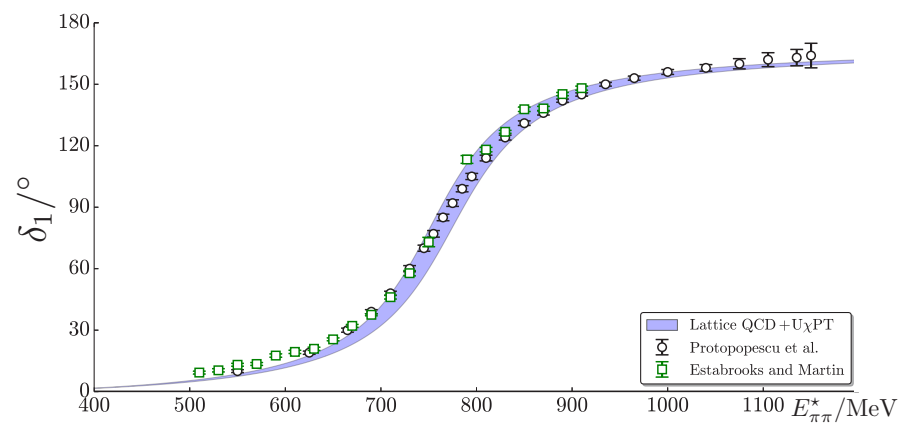

Figure 3. In blue is the isovector $\pi \pi$ scattering phase shift obtained in Ref. [12] by extrapolating results from Ref. [9] using $\mathrm{U} \chi \mathrm{PT}$ as is discussed in the text. This is compared to the experimental data [53-56].

done in a systematic fashion, without ignoring classes of diagrams and using an order of 10-30 operators for each irrep, was in Ref. [52] using two values of the light quark masses corresponding to $m_{\pi}=236,391 \mathrm{MeV}{ }^{6}$

By restricting our attention to energies below the $K \bar{K}$ threshold, Fig. 2 shows the resulting values of the $p \cot \delta$ (proportional to the real part of the inverse of the scattering amplitude) for these two values of $m_{\pi}$. These are compared to the experimental/phenomenological values [17, 53-56]. Although a chiral extrapolation was not performed ${ }^{7}$, one observes a natural trend of these amplitudes as a function of the quark mass.

Having obtained the amplitudes, one can proceed to explore the pole content. For the heavier one finds a real pole below thresholds on the physical sheet, while for the lighter quark mass one finds a complex-valued pole on the second sheet above threshold. This is the manifestation of the $\sigma$ resonance for these quark masses. Once again, from Fig. 2 one finds a natural trajectory between these two points to the manifestation of the broad sigma in nature $[17,57,58]$. Furthermore, from the residue of the amplitudes one can obtain the $\sigma \rightarrow \pi \pi$ coupling, which at this stage seems to have no to mild quark-mass dependence. Naturally, further investigation is needed, but this was the first evidence of the $\sigma$ from lattice QCD.

\subsubsection{Chiral extrapolations}

To this day, most calculations of resonances are being done using unphysically heavy values of the quark masses. The reason for this is multifold. With an increase in Wick contraction and the need of a order of magnitude increase in the basis of operators used, the computational cost of multiparticle observables far outweighs that of single-particle quantities. One can mitigate this cost by restricting to heavier quark masses. As we approach the physical point, we will need to increase the volume in order to keep $m_{\pi} L \sim 4$. This dramatically increases the density of states in the vicinity where we aim to determine the spectrum. This can be tackled with an even larger basis operators. An equally challenging problem emerges due to the fact that the energy cost for producing pion decreases as we approach the chiral limit. This would mean that in order to scan higher energies, where three or more particles can go on-shell, we would need a generalization of Eq. 3 (I come back to this in Sec. 5).

While we proceed to tackle each one of these challenges, we can proceed to consider performing chiral extrapolations of resonant properties obtained at unphysically heavy quark masses. The natural tool for doing this would be chiral perturbation theory ( $\chi$ PT), but at a finite order $\chi$ PT does not exhibit a pole structure $[65,66]$. As a result, it is not suitable for describing resonance or bound states. A technique that enhances the range of applicability of the $\chi \mathrm{PT}$ is the so called the Inverse

\footnotetext{
${ }^{6}$ In Sec. 3.3, I discuss I discuss a recent extension of this work to higher energies for the $m_{\pi}=391 \mathrm{MeV}$ ensembles [63].

${ }^{7}$ See Ref. [64] for first attempts to extrapolate these amplitudes.
} 
Amplitude Method [67-69]. The argument goes as follows. Let us impose force unitarity to be exact at all order in the chiral expansion. In other words, let the scattering amplitude satisfy Eq. 1

$$
\mathcal{M}_{U \chi P T}^{-1} \equiv \operatorname{Re}\left(\mathcal{M}_{\chi P T}^{-1}\right)-i \frac{1}{16 \pi} \frac{2 q^{\star}}{\sqrt{s}} \Theta\left(s-s_{0}\right)
$$

where $\mathcal{M}_{\chi P T}$ is the standard amplitude obtained using $\chi \mathrm{PT}$ and $\mathcal{M}_{U \chi} P T$ is the unitarized amplitude. Going to the next-to-leading order (NLO) in the chiral expansion, and writing $\mathcal{M}_{\chi P T}$ in terms of $\mathcal{M}_{\mathrm{LO}}$ and $\mathcal{M}_{\mathrm{NLO}}$, we find

$$
\mathcal{M}_{\mathrm{U} \chi \mathrm{PT}}=\mathcal{M}_{\mathrm{LO}} \frac{1}{\mathcal{M}_{\mathrm{LO}}-\mathcal{M}_{\mathrm{NLO}}} \mathcal{M}_{\mathrm{LO}}
$$

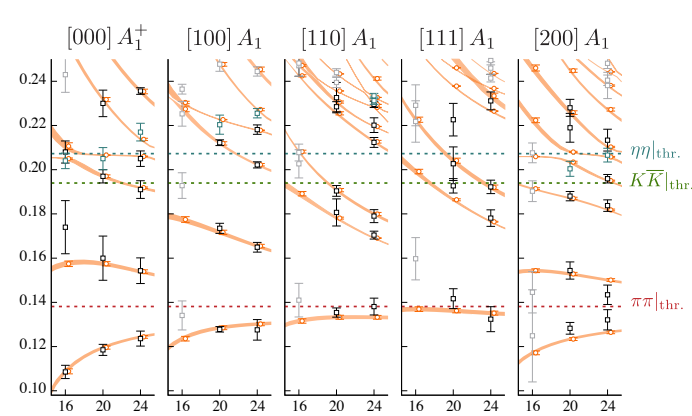

(a)

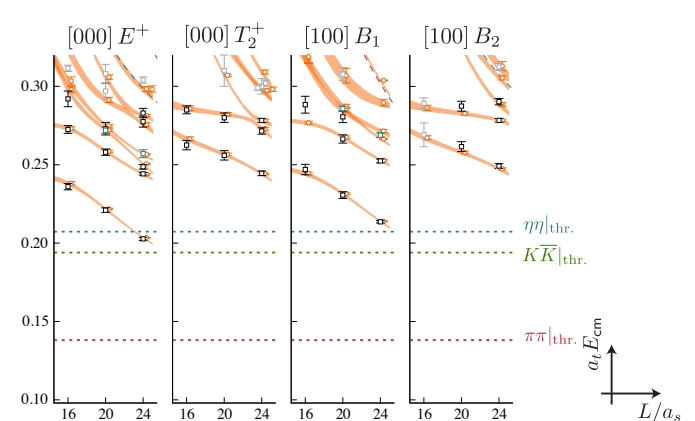

(b)

Figure 4. Shown are the finite-volume spectra obtained in Ref. [63] using $m_{\pi}=391 \mathrm{MeV}$ for the isoscalar $\pi \pi, K \bar{K}, \eta \eta$ sector for irreps that primarily couple to (a) $\ell=0$ and (b) $\ell=2$ partial waves.

At this order, the isovector $\pi \pi$ scattering amplitude is described in terms of two low-energy coefficients (LECs). The same LECs describe both the quark-mass and energy dependence, which are correlated within $\chi \mathrm{PT}$. Exploiting this correlation, Ref. [12] used NLO U $\chi \mathrm{PT}$ to fit the spectra obtained in Ref. [9] (shown in Fig. 1). The resulting amplitude extrapolated to the physical point is shown in Fig. 3, where one sees agreement with experiment [53-56]. Having the amplitude one can proceed to analytically continue and find the $\rho$ pole, shown in Fig. 1. In the same figure one sees agreement with poles obtained by using dispersive techniques to fit experimental data [13-18].

\subsection{Coupled-channel systems: $\pi \pi, K \bar{K}$}

The large portion of resonances lie above multiple open thresholds. In order to study these on the lattice, it is necessary to generalize the idea set forth by Lüscher to any number of open channels. This has been done for energies where only two-particle states can go on-shell [23, 32-34, 37] and the resulting quantization condition has the same form as Eq. 3. Here a problem that I previously glossed over is evident. Equation 3 does not provide a one-to-one mapping between the finite-volume spectrum and infinite-volume scattering amplitude. Instead, a given given energy is related to an infinite number of partial wave amplitudes. This is even true when only one channel is kinematically open. The first time this mixing due to the reduction of rotational symmetry was disentangled in a lattice QCD calculation was in the $I=2 \pi \pi$ channel [70]. The way this was achieved was by using a parametrization for the scattering amplitude. Given some set of parameters, one can use Eq. 3 to predict the finite-volume spectrum which can then directly compared to the spectrum obtained from 


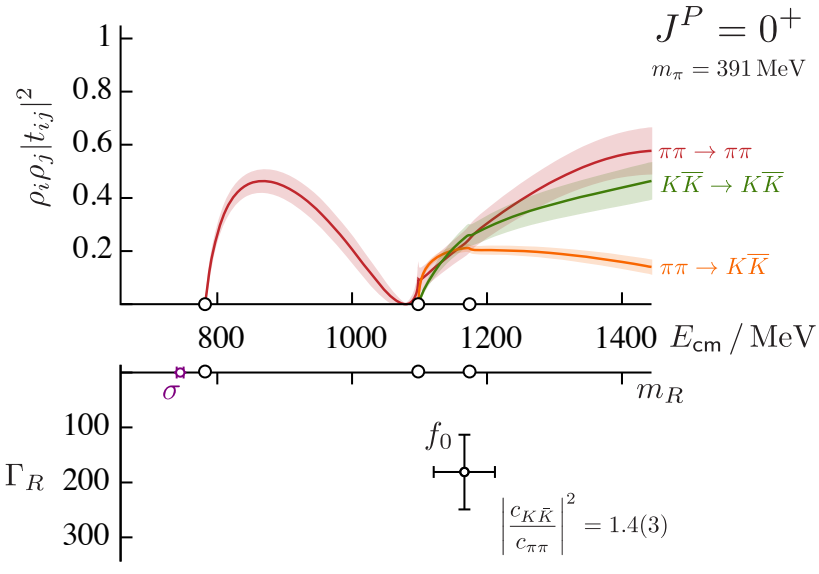

Figure 5. In the top panel are shown the modulus square of the three components of the $\mathrm{S}$-wave scattering amplitude $(t$ is proportional to $\mathcal{M}$ appearing Eq. 1) describing the coupled $\pi \pi, K \bar{K}$ isoscalar channels obtained in Ref. [63] using $m_{\pi}=391 \mathrm{MeV}$. Bottom panel shows the poles of the $\sigma$ and $f_{0}$ resonances.

the two-point correlation function. One can adopt this procedure even when the amplitudes are not diagonal, i.e., when channels are coupled. ${ }^{8}$

These ideas were first put into practice for coupled channels in the study of $K \pi, K \eta$ scattering in the isodoublet channel using $m_{\pi}=391 \mathrm{MeV}[72,73]$. At these values of the quark masses, these two channels were practically decoupled. Similarly in Ref. [9] $\pi \pi$ and $K \bar{K}$ were found to be practically decoupled in the channel of the $\rho$-resonances. The first strongly coupled calculations were performed in Refs. [74, 75], and most recently this was put into practice in the isoscalar sector [63]. This study was an extension of the isoscalar calculations of Ref. [52], discussed in Sec. 3.2.

Reference [63] presented the finite-volume spectrum of $\pi \pi, K \bar{K}$, and $\eta \eta$ in the isoscalar sector for the $m_{\pi}=391 \mathrm{MeV}$ ensembles. The resulting spectrum, including a fit to it using one of a large range of parametrizations is shown in Fig. 4. Although the spectrum is interesting on its own, the more interesting quantity to consider is the scattering amplitude. In Figures 5 and 6 show the different components of the scattering amplitude, ignoring weak channels where the $\eta \eta$ is present, in the $\ell=0$ and $\ell=2$ partial waves respectively. Some prominent features of the $S$-wave amplitude is the fact that $\pi \pi$ and $K \bar{K}$ are very strongly coupled, similar to what is known phenomenologically. In addition to finding evidence of the $\sigma$, discussed above, these amplitudes show a clear signal of the resonance near the $K \bar{K}$ threshold. The location and relative coupling to the two channels is shown in Fig. 5. Figure 6 shows the $\ell=2$ partial wave amplitudes. As is evident, in this partial wave the two channels are practically decoupled. Also, one sees two clear bump-like structures. By exploring the behavior of these amplitudes in the complex plane, one finds the two resonances depicted in Fig. 6.

\section{Electroweak process from lattice QCD}

In general, one would like study electroweak reactions involving scattering states in the initial and/or final state. This adds yet another source of complexity. If we are only interested in studying reactions where the effects of the electroweak sector are perturbative, the most natural means to access information of these reactions is by evaluating three-point functions with single insertions of the external electroweak current. In doing so, one can in principle extract finite-volume matrix elements

\footnotetext{
${ }^{8}$ For parallel/alternative efforts for extracting coupled-channel scattering amplitudes presented in this conference see [71].
} 


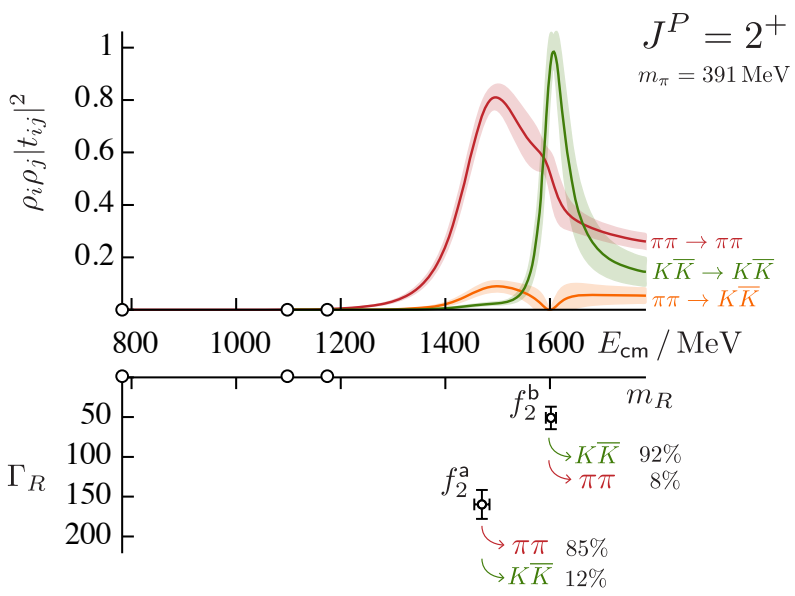

Figure 6. The top panel shows the same as Fig. 5 but for the $\ell=2$ partial waves. Bottom panel shows the poles of the two lowest lying resonances in this channel.

of these currents,

$$
C_{a b}^{3 p t .}\left(t_{f}, \mathbf{P}_{f} ; \mathbf{P}_{i}, t_{i}\right) \equiv\left\langle 0\left|O_{b}\left(t_{f}, \mathbf{P}_{f}\right) \mathcal{J}(0) O_{a}^{\dagger}\left(t_{i}, \mathbf{P}_{i}\right)\right| 0\right\rangle \propto\left\langle E_{n_{f}}, \mathbf{P}_{f}, L|\mathcal{J}(0)| E_{n_{i}}, \mathbf{P}_{i}, L\right\rangle+\cdots,
$$

where $\left|E_{n_{i}}, \mathbf{P}_{i}, L\right\rangle$ explicitly denote the finite-volume states and the ellipses denote contribution associated with overlap with other states. Being able to extract the matrix elements of a given state (even the ground state) has been a historical challenge. Recently, it has been demonstrated that in practice one can use the same techniques for extracting the excited state spectrum to also obtaining the matrix elements of the corresponding states [76].

Having then, in principle, determined such a matrix element, we must then understand how this matrix elements must be interpreted. When the initial and final states are stable under the strong interactions, these matrix elements are exponentially close to their infinite-volume counterparts

$$
\left\langle E_{n_{f}}, \mathbf{P}_{f}, L|\mathcal{J}(0)| E_{n_{i}}, \mathbf{P}_{i}, L\right\rangle=\left\langle E_{n_{f}}, \mathbf{P}_{f}, \infty|\mathcal{J}(0)| E_{n_{i}}, \mathbf{P}_{i}, \infty\right\rangle+O\left(e^{-m_{\pi} L}\right) .
$$

When the initial and/or final states are near or above a threshold, the relationship between this and their infinite-volume counterparts are not obvious.

\subsection{Interpreting $1 \rightarrow 2$ matrix elements}

In recent years there has been substantial effort in developing the necessary formalism for studying matrix elements involving two particles in the initial and/or final state. These ideas largely stem from work developed by Lellouch and Lüscher explaining how $K \rightarrow \pi \pi$ weak decays can be accessed from the lattice [77]. These ideas have since been generalized to increasingly complex $0 \rightarrow 2$ [78, 79], and $1 \rightarrow 2$ reactions [26, 27, 33, 80-82]. In Ref. [83] the most general results for studying $0 \rightarrow 2$ and $1 \rightarrow 2$ transitions from finite volume matrix elements was presented. Although it turns out that these are closely, here I only discuss the latter.

Consider a current coupling a finite volume state $\left|E_{n}, \mathbf{P}^{\prime} L\right\rangle$ to another state $\left|E_{0}^{(1)}, \mathbf{P}, L\right\rangle$. Here we restrict our attention to the scenario where the latter correspond to a QCD stable state and where the volumes satisfy $m_{\pi} L \gg 1$. This means we can assume $\left|E_{0}^{(1)}, \mathbf{P}, L\right\rangle=\left|E_{0}^{(1)}, \mathbf{P}, \infty\right\rangle$ up to negligible contributions. Let $\left[E_{n}^{2}-\mathbf{P}^{\prime 2}\right]^{1 / 2}$ be the $n t h$ state in the tower of states and we let lie above any number of two-particle thresholds but below all thresholds where three or more particles can go on-shell. 


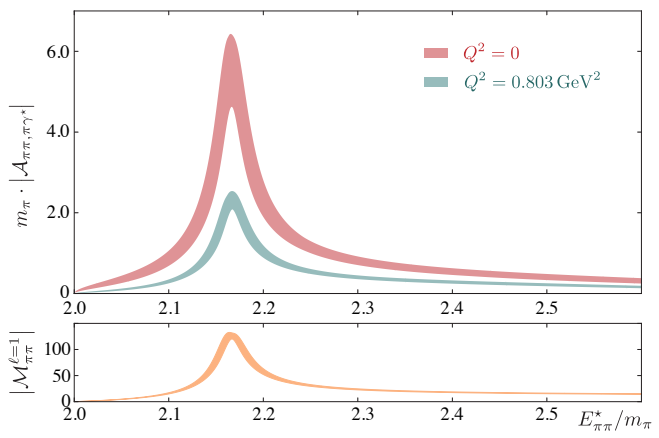

(a)

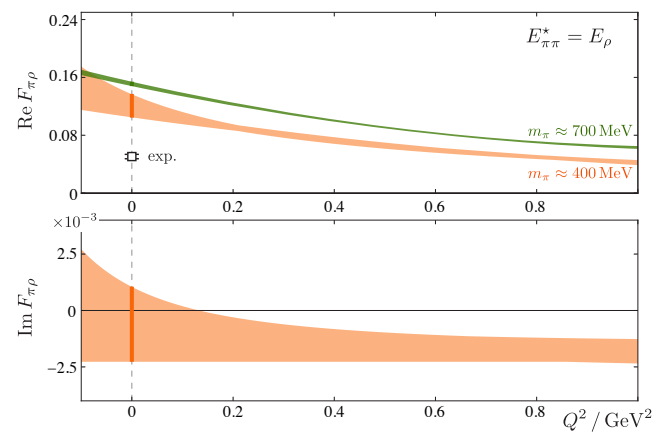

(b)

Figure 7. (a) Top panel shows the $\pi \gamma^{\star} \rightarrow \pi \pi$ amplitude using $m_{\pi}=391 \mathrm{MeV}$ as a function of the $\pi \pi$ energy [84, 85]. Bottom panel shows the elastic $\pi \pi$ amplitude for the same ensemble [8]. (b) Shown is the real and imaginary components of the $\pi \rightarrow \rho$ form factor form $m_{\pi}=700 \mathrm{MeV}$ [76] and $m_{\pi}=391 \mathrm{MeV}[84,85]$.

Finally, we label $\mathcal{H}$ to be the infinite-volume amplitude coupling the initial and final states. This amplitude is non-perturbative in the strong interactions but perturbative in the electroweak dynamics. We can write this amplitude as a vector in the degrees of freedom of the system, namely the partial waves of the two-particle state and the number of open channels open. With this, we can write a compact relationship between the finite-volume matrix element and the infinite-volume amplitude [83]

$$
L^{3}\left|\left\langle E_{n}, \mathbf{P}^{\prime} L|\mathcal{J}(0)| E_{0}^{(1)}, \mathbf{P}, \infty\right\rangle\right|=\frac{1}{\sqrt{2 E_{0}^{(1)}}} \sqrt{\mathcal{H}^{T}\left[\mathcal{R}\left(E_{n}, \mathbf{P}\right)\right] \mathcal{H}},
$$

where $\mathcal{R}$ is the residue of the finite-volume two-particle propagator,

$$
\mathcal{R}\left(E_{n}, \mathbf{P}\right) \equiv \lim _{E \rightarrow E_{n}}\left[\left(E-E_{n}\right) \frac{1}{F^{-1}(P, L)+\mathcal{M}(s)}\right] .
$$

Here $F$ and $\mathcal{M}$ are the same that appear in the quantization condition of the two-particle spectrum, Eq. 3. In general, this is a matrix in the degrees of freedom of the two-particle channel. In the limit that a single channel is open and partial waves do not mix, only one component of this matrix would contribute, which is commonly referred to in the literature as the Lellouch-Lüscher factor. ${ }^{9}$

This equation can be explained as follows. Given the spectrum, one determine $F, \mathcal{M}$, and consequently $\mathcal{R}$. Next, one must determine the finite-volume matrix element using the standard three-point functions (arguably the most challenging task present). Once these are known one can use the mapping defined by Eq. 9 to then obtained the desired infinite-volume amplitude $\mathcal{H}$.

\section{$4.2 \pi \gamma^{\star} \rightarrow \pi \pi$ and the $\pi \rightarrow \rho$ form factor}

The formalism summarized above represents some of the first steps toward the lattice QCD community being able say anything about the electromagnetic resonant processes. One of the first examples of this was the $\gamma^{\star} \rightarrow \pi \pi$ amplitude obtained for the first time in Ref. [79] and now being explored

\footnotetext{
${ }^{9}$ Although here we assume periodic symmetric volume, Ref. [83] presents a result that holds when the volume is asymmetric with arbitrary twisted boundaries conditions.
} 
by others [86]. These studies are partially motivated by the important role this amplitude plays in constraining the dominant hadronic contribution to anomalous magnetic moment of the muon.

A slightly more complicated process is $\gamma^{\star} \pi \rightarrow \pi \pi$, which mimics the electro- and photoproduction reactions explored in, for example, the Thomas Jefferson National Accelerator Facility (JLab). Given the important role played in the low-energy manifestation of QCD, it should not come as a surprised that this reaction plays an important role in a variety of processes, ranging from the chiral anomaly to the anomalous magnetic moment of the muon (see Refs. [87, 88] for recent phenomenological studies of this amplitude).

The first study lattice QCD study of $\gamma^{\star} \pi \rightarrow \pi \pi$ was performed in Refs. [84, 85]. This calculation was done using the same $m_{\pi}=391 \mathrm{MeV}$ ensembles discussed above. In this study the $\gamma^{\star} \pi \rightarrow \pi \pi$ amplitude was determine for a range of energy of the $\pi \pi$ state and virtuality of the photon. A global fit of this amplitude projected onto two virtualities and a range of energies is shown in Fig. 7. One sees a clear manifestation of the $\rho$ resonance, which coincides with its manifestation in the elastic $\pi \pi$ amplitude.

By exploring the energy dependence of the this amplitude, one may obtain the $\pi \rightarrow \rho$ form factor. This can be defined in terms of the residue of the the amplitude at the $\rho$ pole. This form factor is shown in Fig. 7 as a function of the virtuality for two values of the pion mass, one where the $\rho$ is stable [76] and another when it is unstable [84, 85].

\section{Future prospects}

Here I have attempted to give overview of the progress made in the studies of resonances on the lattice (for a more detailed review see Ref. [6]). I aimed to focus on ideas where there has been at least one application in a lattice calculation. Here I discuss what I believe might be three main areas of growth in future studies of resonance properties from lattice QCD.

Need for dispersive analysis: In right panel of Fig. 2 we see the first determination of the $\sigma$ resonance pole from lattice QCD. [52]. For the heavier ensemble, this turned to be a bound state. For the lighter ensemble, the $\sigma$ closely mirrors the experimental one, meaning it appears as a broad resonance. In practice, this means that one must analytically continue amplitudes constrained on the real axis far into the complex plane. This is challenging problem, which explains why the mass of the $\sigma$ has historically had such large systematic error. By choosing a different parametrization, one obtains a different pole. This also explains why for the $m_{\pi}=236 \mathrm{MeV}$ ensemble one sees a scatter of points in Fig. 2. Phenomenologically, it is now well known that this systematic error can be greatly reduced by using a dispersive analysis of experimental scattering data $[17,57,58]$. This example clearly shows the need for a more sophisticated analysis of finite-volume spectra, where similarly ideas to those being used in modern-day amplitude analysis are adopted. Two other examples that illustrate the need for more sophisticated amplitude analysis are the coupled-channel systems (discussed in Sec. 3.3) and three-body systems (discussed below)

Electroweak $2 \rightarrow 2$ processes and elastic form factors: Two classes of processes that are apparently accessible from lattice QCD but experimentally inaccessible are electroweak $2 \rightarrow 2$ processes and three-body scattering. Let me first discuss the former. These are amplitudes where a two-particle couples to an external current and then results in another or the same two-particle state. These amplitudes, which are challenging to access experimentally, have been proposed in the literature for, for example, evaluating electromagnetic [90] and scalar [91] form factors of resonances.

The idea for determining these amplitudes from two-body matrix elements was first proposed in Refs. [32, 92, 93]. These studies explained that indeed for some classes of currents and hadrons, one can indeed access infinite-volume observables from finite-volume matrix elements. Reference [89] 


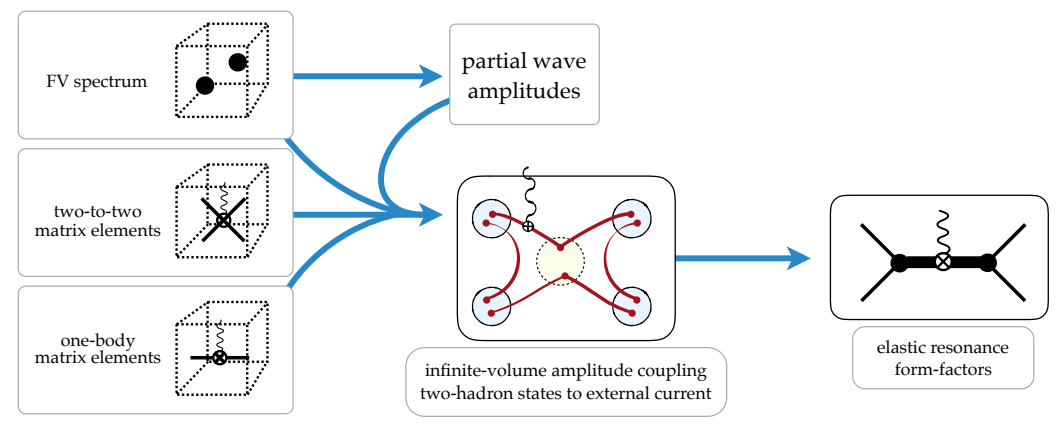

Figure 8. Shown is a schematic workflow for obtaining resonance form factors from lattice QCD observables [89]. Details are explained in the text.

was the first to derive a model-independent relation between such matrix elements and the corresponding amplitude.

The $2 \rightarrow 2$ amplitude, $\mathcal{W}$, has kinematic singularities associated with intermediate particles going on-shell. The matrix elements are related to these amplitudes where the kinematic singularities have been exactly removed, resulting in the so-called divergence free amplitude, $\mathcal{W}_{\mathrm{df}}$. This can be related to the finite-volume matrix element using the same $\mathcal{R}$ defined in Eq. 10,

$$
\left|\left\langle E_{n_{f}}, \mathbf{P}_{f}, L|\mathcal{J}(0)| E_{n_{i}}, \mathbf{P}_{i}, L\right\rangle\right|^{2}=\frac{1}{L^{6}} \operatorname{Tr}\left[\mathcal{R} \mathcal{W}_{L, \mathrm{df}} \mathcal{R} \mathcal{W}_{L, \mathrm{df}}\right],
$$

$W_{L, \mathrm{df}}$ is related to the aforementioned $\mathcal{W}_{\mathrm{df}}$,

$$
\mathcal{W}_{L, \mathrm{df}} \equiv \mathcal{W}_{\mathrm{df}}+\mathcal{M} \mathcal{G} \mathcal{M}
$$

and $\mathcal{G}$ is a new finite-volume function that dependence on the spectrum and the single particle matrix elements.

This relation is similar to that appearing in Eq. 9, and some the quantities needed are the same, namely the spectrum and $\mathcal{R}$. The one main difference is this new finite-volume function $G$. As was stated, this depend on the single particle matrix elements. This refers to the matrix elements of the constituents of the two-particle state. For example, if one would want to use this to study the $\pi \pi \mathcal{J} \rightarrow \pi \pi$ amplitude, one would also need to determine the $\pi \mathcal{J} \rightarrow \pi$, to then use Eq. 11. Figure 8 summarizes the different ingredients needed to obtain the desired $2 \rightarrow 2$ amplitude. Once $W_{\mathrm{df}}$ is obtained, one can proceed to analytically continue onto the resonance poles of the incoming and final states, to extract elastic and/or inelastic resonance form factors. This is all to say that it is not unfathomable to, in the not so distant future, study the structure of unstable states.

Three particles or more: All of the lattice QCD calculations of resonances have been restricted to states that couple solely to two-particles. This is in large part due to the fact that we do not yet understand, in general, how to extract three-body observables from finite-volume spectra. In recent years, there has been tremendous amount of progress with the aim of circumventing this problem [94104]. ${ }^{10}$ Presently, the most general result for studying systems coupling to three-particles or less can be found in Ref. [101, 104],

$$
\operatorname{det}\left[1+\left(\begin{array}{cc}
F_{2} & 0 \\
0 & F_{3}
\end{array}\right)\left(\begin{array}{cc}
\mathcal{K}_{2} & \mathcal{K}_{23} \\
\mathcal{K}_{32} & \mathcal{K}_{\mathrm{df}, 3}
\end{array}\right)\right]=0
$$

This quantization condition holds for energies where two- and three-particle states can go on-shell, and it gives a relationship between the spectrum and the infinite-volume $\mathrm{K}$ matrix. Unitarity relates the K matrix to the scattering amplitude, and Ref. [104] gives an exact relationship between these.

\footnotetext{
${ }^{10}$ For development in 1+1 dimensional systems see [105-107].
} 
Unlike the two-particle analogue, Eq. 3, this is not quite general. To this date all work involving three-particle states have assumed the individual particles to be identical bosons. This is a mild assumption, that could be easily removed. More restrictive is the fact that this equation only holds for kinematic regions where the two-body $\mathrm{K}$ matrix is non singular. This is a technical restriction that has not yet been surpassed. Once this formalism is complete, it is not hard to imagine being able to extend these ideas to study $1 \rightarrow 3$ transitions. This would be relevant for, for example, $K \rightarrow \pi \pi \pi$ weak decays and $N \gamma^{\star} \rightarrow N^{\star} \rightarrow N \pi \pi$ transitions.

\section{Final remarks}

We are entering an exciting era in hadronic physics. In the last few year, we have witnessed a tremendous amount of progress from the lattice QCD community in many fronts. One subfield that has surpassed its original expectations is the study of few-hadron resonant as well as non-resonant reactions. This field has been partly guided by formal ideas, some of which have been set forth over a quarter of a century ago, others that are being actively developed. But these formal concepts would be useless without the algorithmic and numerical developments.

Arguably, the algorithmic developments have surpassed the expectations of the more formal community, which has now ignited a flurry of theoretical activity to explore processes that were previously believe to be inaccessible. This has in turn decreased the time period where ideas are being set out and when they put into practice. This is all to say that now is a perfect time to be working in these ideas, to develop them and immediately test them out in phenomenologically relevant calculations.

Finally, it is worth emphasizing that there is lots of room for growth. As I tried to emphasize in the previous section, the challenges we are presently encountering are ones that could benefit from the attention of theoreticians with a diverse set of skills. Unarguably the most important discoveries for this field lie ahead. 


\section{References}

[1] K. Erkelenz, Phys. Rept. 13, 191 (1974)

[2] F. Hoyle, Astrophys. J. Suppl. 1, 121 (1954)

[3] G.K.C. Cheung, C.E. Thomas, J.J. Dudek, R.G. Edwards (Hadron Spectrum) (2017), 1709.01417

[4] C. Hughes, E. Eichten, C.T.H. Davies, Submitted to: Phys. Rev. D (2017), 1710.03236

[5] R.F. Lebed, R.E. Mitchell, E.S. Swanson, Prog. Part. Nucl. Phys. 93, 143 (2017), 1610. 04528

[6] R.A. Briceno, J.J. Dudek, R.D. Young (2017), 1706. 06223

[7] V. Gribov, Strong interactions of hadrons at high energies: Gribov lectures on theoretical physics, Cambridge monographs on particle physics, nuclear physics, and cosmology (Cambridge Univ. Press, Cambridge, 2008), https://cds . cern.ch/record/1186219

[8] J.J. Dudek, R.G. Edwards, C.E. Thomas (Hadron Spectrum), Phys. Rev. D87, 034505 (2013), [Erratum: Phys. Rev.D90,no.9,099902(2014)], 1212.0830

[9] D.J. Wilson, R.A. Briceno, J.J. Dudek, R.G. Edwards, C.E. Thomas, Phys. Rev. D92, 094502 (2015), 1507.02599

[10] H.W. Lin et al. (Hadron Spectrum), Phys. Rev. D79, 034502 (2009), 0810. 3588

[11] J.J. Dudek, R.G. Edwards, P. Guo, C.E. Thomas (Hadron Spectrum), Phys. Rev. D88, 094505 (2013), 1309.2608

[12] D.R. Bolton, R.A. Briceno, D.J. Wilson, Phys. Lett. B757, 50 (2016), 1507.07928

[13] P. Masjuan, J. Ruiz de Elvira, J.J. Sanz-Cillero, Phys. Rev. D90, 097901 (2014), 1410. 2397

[14] B. Ananthanarayan, G. Colangelo, J. Gasser, H. Leutwyler, Phys. Rept. 353, 207 (2001), hep-ph/0005297

[15] G. Colangelo, J. Gasser, H. Leutwyler, Nucl.Phys. B603, 125 (2001), hep-ph/0103088

[16] Z.Y. Zhou, G.Y. Qin, P. Zhang, Z. Xiao, H.Q. Zheng, N. Wu, JHEP 02, 043 (2005), hep-ph/0406271

[17] R. Garcia-Martin, R. Kaminski, J.R. Pelaez, J. Ruiz de Elvira, Phys. Rev. Lett. 107, 072001 (2011), 1107. 1635

[18] P. Masjuan, J.J. Sanz-Cillero, Eur. Phys. J. C73, 2594 (2013), 1306. 6308

[19] M. Luscher, Commun. Math. Phys. 105, 153 (1986)

[20] M. Luscher, Nucl. Phys. B354, 531 (1991)

[21] K. Rummukainen, S.A. Gottlieb, Nucl. Phys. B450, 397 (1995), hep-lat/9503028

[22] X. Feng, X. Li, C. Liu, Phys. Rev. D70, 014505 (2004), hep-lat/0404001

[23] S. He, X. Feng, C. Liu, JHEP 07, 011 (2005), hep-lat/0504019

[24] P.F. Bedaque, Phys. Lett. B593, 82 (2004), nucl-th/0402051

[25] C. Liu, X. Feng, S. He, Int.J.Mod.Phys. A21, 847 (2006), hep-lat/0508022

[26] C.h. Kim, C.T. Sachrajda, S.R. Sharpe, Nucl. Phys. B727, 218 (2005), hep-lat/0507006

[27] N.H. Christ, C. Kim, T. Yamazaki, Phys. Rev. D72, 114506 (2005), hep-lat/0507009

[28] M. Lage, U.G. Meiner, A. Rusetsky, Phys. Lett. B681, 439 (2009), 0905.0069

[29] V. Bernard, M. Lage, U.G. Meiner, A. Rusetsky, JHEP 01, 019 (2011), 1010.6018

[30] Z. Fu, Phys. Rev. D85, 014506 (2012), 1110.0319

[31] L. Leskovec, S. Prelovsek, Phys. Rev. D85, 114507 (2012), 1202 . 2145

[32] R.A. Briceno, Z. Davoudi, Phys. Rev. D88, 094507 (2013), 1204.1110

[33] M.T. Hansen, S.R. Sharpe, Phys. Rev. D86, 016007 (2012), 1204.0826

[34] P. Guo, J. Dudek, R. Edwards, A.P. Szczepaniak, Phys. Rev. D88, 014501 (2013), 1211.0929 
[35] N. Li, C. Liu, Phys. Rev. D87, 014502 (2013), 1209. 2201

[36] R.A. Briceno, Z. Davoudi, T.C. Luu, M.J. Savage, Phys. Rev. D89, 074509 (2014), 1311.7686

[37] R.A. Briceno, Phys. Rev. D89, 074507 (2014), 1401. 3312

[38] M. Lüscher, U. Wolff, Nucl. Phys. B339, 222 (1990)

[39] J. Foley, K. Jimmy Juge, A. O'Cais, M. Peardon, S.M. Ryan, J.I. Skullerud, Comput. Phys. Commun. 172, 145 (2005), hep-lat/0505023

[40] M. Peardon, J. Bulava, J. Foley, C. Morningstar, J. Dudek, R.G. Edwards, B. Joo, H.W. Lin, D.G. Richards, K.J. Juge (Hadron Spectrum), Phys. Rev. D80, 054506 (2009), 0905.2160

[41] B. Blossier, M. Della Morte, G. von Hippel, T. Mendes, R. Sommer, JHEP 04, 094 (2009), 0902.1265

[42] J.J. Dudek, R.G. Edwards, M.J. Peardon, D.G. Richards, C.E. Thomas, Phys. Rev. Lett. 103, 262001 (2009), 0909.0200

[43] C. Morningstar, J. Bulava, J. Foley, K.J. Juge, D. Lenkner, M. Peardon, C.H. Wong, Phys. Rev. D83, 114505 (2011), 1104.3870

[44] J.J. Dudek, R.G. Edwards, M.J. Peardon, D.G. Richards, C.E. Thomas, Phys. Rev. D82, 034508 (2010), 1004.4930

[45] L. Liu, G. Moir, M. Peardon, S.M. Ryan, C.E. Thomas, P. Vilaseca, J.J. Dudek, R.G. Edwards, B. Joo, D.G. Richards (Hadron Spectrum), JHEP 07, 126 (2012), 1204.5425

[46] J.J. Dudek, R.G. Edwards, Phys. Rev. D85, 054016 (2012), 1201.2349

[47] J.J. Dudek, Phys. Rev. D84, 074023 (2011), 1106. 5515

[48] R.G. Edwards, J.J. Dudek, D.G. Richards, S.J. Wallace, Phys. Rev. D84, 074508 (2011), 1104.5152

[49] J.J. Dudek, R.G. Edwards, B. Joo, M.J. Peardon, D.G. Richards, C.E. Thomas, Phys. Rev. D83, 111502 (2011), 1102.4299

[50] C. Michael, Nucl. Phys. B259, 58 (1985)

[51] C.E. Thomas, R.G. Edwards, J.J. Dudek, Phys. Rev. D85, 014507 (2012), 1107 . 1930

[52] R.A. Briceno, J.J. Dudek, R.G. Edwards, D.J. Wilson, Phys. Rev. Lett. 118, 022002 (2017), 1607.05900

[53] S.D. Protopopescu, M. Alston-Garnjost, A. Barbaro-Galtieri, S.M. Flatte, J.H. Friedman, T.A. Lasinski, G.R. Lynch, M.S. Rabin, F.T. Solmitz, Phys. Rev. D7, 1279 (1973)

[54] B. Hyams et al., Nucl. Phys. B64, 134 (1973)

[55] G. Grayer et al., Nucl. Phys. B75, 189 (1974)

[56] P. Estabrooks, A.D. Martin, Nucl. Phys. B79, 301 (1974)

[57] J.R. Pelaez, Phys. Rept. 658, 1 (2016), 1510.00653

[58] I. Caprini, G. Colangelo, H. Leutwyler, Phys. Rev. Lett. 96, 132001 (2006), hep-ph/0512364

[59] C. Alexandrou, L. Leskovec, S. Meinel, J. Negele, S. Paul, M. Petschlies, A. Pochinsky, G. Rendon, S. Syritsyn, Phys. Rev. D96, 034525 (2017), 1704 . 05439

[60] J. Bulava, B. Fahy, B. Horz, K.J. Juge, C. Morningstar, C.H. Wong, Nucl. Phys. B910, 842 (2016), 1604.05593

[61] J. Bulava, B. Horz, C. Morningstar, Multi-hadron spectroscopy in a large physical volume (2017), 1710.04545, https://inspirehep.net/record/1630476/files/arXiv: 1710 . Q4545.pdf

[62] S. Aoki et al. (CS), Phys. Rev. D84, 094505 (2011), 1106.5365

[63] R.A. Briceno, J.J. Dudek, R.G. Edwards, D.J. Wilson (2017), 1708.06667

[64] M. Doring, B. Hu, M. Mai (2016), 1610. 10070 
[65] S. Weinberg, Phys. Rev. Lett. 17, 616 (1966)

[66] J. Gasser, H. Leutwyler, Phys. Lett. B125, 325 (1983)

[67] J.A. Oller, E. Oset, J.R. Pelaez, Phys. Rev. Lett. 80, 3452 (1998), hep-ph/9803242

[68] J.A. Oller, E. Oset, J.R. Pelaez, Phys. Rev. D59, 074001 (1999), [Erratum: Phys. Rev.D75,099903(2007)], hep-ph/9804209

[69] A. Gomez Nicola, J.R. Pelaez, Phys. Rev. D65, 054009 (2002), hep-ph/0109056

[70] J.J. Dudek, R.G. Edwards, C.E. Thomas, Phys. Rev. D86, 034031 (2012), 1203.6041

[71] R. Brett, J. Bulava, J. Fallica, A. Hanlon, B. Horz, C. Morningstar, B. Singha, Scattering from finite-volume energies including higher partial waves and multiple decay channels (2017), 1710.04169, https://inspirehep.net/record/1629976/files/arXiv: 1710.04169. $\operatorname{pdf}$

[72] J.J. Dudek, R.G. Edwards, C.E. Thomas, D.J. Wilson (Hadron Spectrum), Phys. Rev. Lett. 113, 182001 (2014), 1406.4158

[73] D.J. Wilson, J.J. Dudek, R.G. Edwards, C.E. Thomas, Phys. Rev. D91, 054008 (2015), 1411.2004

[74] J.J. Dudek, R.G. Edwards, D.J. Wilson (Hadron Spectrum), Phys. Rev. D93, 094506 (2016), 1602.05122

[75] G. Moir, M. Peardon, S.M. Ryan, C.E. Thomas, D.J. Wilson, JHEP 10, 011 (2016), 1607.07093

[76] C.J. Shultz, J.J. Dudek, R.G. Edwards, Phys. Rev. D91, 114501 (2015), 1501.07457

[77] L. Lellouch, M. Luscher, Commun. Math. Phys. 219, 31 (2001), hep-lat/0003023

[78] H.B. Meyer, Phys. Rev. Lett. 107, 072002 (2011), 1105. 1892

[79] X. Feng, S. Aoki, S. Hashimoto, T. Kaneko, Phys. Rev. D91, 054504 (2015), 1412 . 6319

[80] H.B. Meyer (2012), 1202.6675

[81] A. Agadjanov, V. Bernard, U.G. Meißner, A. Rusetsky, Nucl. Phys. B886, 1199 (2014), 1405.3476

[82] R.A. Briceño, M.T. Hansen, A. Walker-Loud, Phys. Rev. D91, 034501 (2015), 1406. 5965

[83] R.A. Briceño, M.T. Hansen, Phys. Rev. D92, 074509 (2015), 1502 . 04314

[84] R.A. Briceno, J.J. Dudek, R.G. Edwards, C.J. Shultz, C.E. Thomas, D.J. Wilson, Phys. Rev. Lett. 115, 242001 (2015), 1507.06622

[85] R.A. Briceño, J.J. Dudek, R.G. Edwards, C.J. Shultz, C.E. Thomas, D.J. Wilson, Phys. Rev. D93, 114508 (2016), 1604.03530

[86] J. Bulava, B. Horz, B. Fahy, K.J. Juge, C. Morningstar, C.H. Wong, PoS LATTICE2015, 069 (2016), 1511.02351

[87] M. Hoferichter, B. Kubis, M. Zanke (2017), 1710.00824

[88] M. Hoferichter, B. Kubis, D. Sakkas, Phys. Rev. D86, 116009 (2012), 1210. 6793

[89] R.A. Briceño, M.T. Hansen, Phys. Rev. D94, 013008 (2016), 1509.08507

[90] T. Sekihara, T. Hyodo, D. Jido, Phys. Lett. B669, 133 (2008), 0803. 4068

[91] M. Albaladejo, J.A. Oller, Phys. Rev. D86, 034003 (2012), 1205.6606

[92] W. Detmold, M.J. Savage, Nucl. Phys. A743, 170 (2004), hep-lat/0403005

[93] V. Bernard, D. Hoja, U.G. Meiner, A. Rusetsky, JHEP 09, 023 (2012), 1205.4642

[94] K. Polejaeva, A. Rusetsky, Eur. Phys. J. A48, 67 (2012), 1203. 1241

[95] R.A. Briceno, Z. Davoudi, Phys. Rev. D87, 094507 (2013), 1212 . 3398

[96] M.T. Hansen, S.R. Sharpe, Phys. Rev. D92, 114509 (2015), 1504.04248

[97] H.W. Hammer, J.Y. Pang, A. Rusetsky (2017), 1707. 02176 
[98] M. Mai, M. Doring (2017), 1709. 08222

[99] S.R. Sharpe, Phys. Rev. D96, 054515 (2017), 1707.04279

[100] M.T. Hansen, S.R. Sharpe, Phys. Rev. D90, 116003 (2014), 1408. 5933

[101] R.A. Briceno, M.T. Hansen, S.R. Sharpe, PoS LATTICE2016, 115 (2016), 1609.09805

[102] M.T. Hansen, S.R. Sharpe, Phys. Rev. D93, 096006 (2016), [Erratum: Phys. Rev.D96,no.3,039901(2017)], 1602.00324

[103] M.T. Hansen, S.R. Sharpe, Phys. Rev. D95, 034501 (2017), 1609.04317

[104] R.A. Briceno, M.T. Hansen, S.R. Sharpe, Phys. Rev. D95, 074510 (2017), 1701.07465

[105] P. Guo, Phys. Rev. D95, 054508 (2017), 1607.03184

[106] P. Guo, V. Gasparian, Phys. Lett. B774, 441 (2017), 1701.00438

[107] P. Guo, V. Gasparian (2017), 1709.08255 